\title{
El clima organizacional en el emprendimiento sostenible
}

\begin{abstract}
Yamarú del Valle Chirinos Araque ${ }^{1}$ Universidad Católica Luis Amigó yamaru.chirinosar@amigo.edu.co

Víctor Hugo Meriño Córdoba² Universidad Católica Luis Amigó victor.merinoco@amigo.edu.co

Carmen Martínez de Meriño

DOI: https://doi.org/10.21158/01208160.n84.2018.1916

Universidad Nacional Experimental Rafael María Baralt cimartinezunermb@gmail.com
\end{abstract}

Fecha de recepción: 30 de agosto de 2017

Fecha de aprobación: 09 de marzo de 2018

Cómo citar este artículo / To reference this article / Comment citer cet article / Para citar este artigo:

Chirinos Araque, Y.; Meriño Córdoba, V. H. y Martínez de Meriño, C.. (2018). El clima organizacional en el emprendimiento sostenible. Revista EAN, 84, (pp 43 - 61). DOI: https://doi.org/10.21158/01208160.n84.2018.1916

\section{Resumen}

El estudio tiene como objetivo analizar el clima organizacional en el emprendimiento sostenible, con el fin de identificar la influencia del ambiente laboral en el desarrollo del emprendimiento y en el éxito del mismo, donde se hace énfasis en la importancia que tiene para la interacción entre las personas. Se consideraron los enfoques de Drucker (2011), Chirinos \& Pérez (2016), Alcóver, Rico \& Gil (2011), entre otros. La investigación fue orientada desde el paradigma cuantitativo, el tipo de investigación fue descriptiva, transaccional de campo. Los resultados obtenidos indican que el clima organizacional es bajo, incidiendo negativamente en el desarrollo del emprendimiento sostenible, en conclusión, los empleados se sienten insatisfechos con el clima organizacional en su área laboral.

\section{Palabras clave}

Clima organizacional, capital intelectual, emprendimiento sostenible.

\footnotetext{
${ }^{1}$ Licenciada en Administración Industrial, Universidad Nacional Experimental Rafael María Baralt. Doctora en Gestión de la innovación, Universidad Dr. Rafael Belloso Chacín. Investigadora Junior categorizada por Colciencias. Docente- investigador del grupo: GORAS de la Universidad Católica Luis Amigó - Sede Medellín. ORCID: https://orcid.org/0000-0003-04719859

${ }^{2}$ Licenciado en Matemática, Universidad del Zulia. Doctor en Educación, Universidad Dr. Rafael Belloso Chacín. Docenteinvestigador del grupo: GORAS de la Universidad Católica Luis Amigó - Sede Medellín. ORCID: https://orcid.org/00000001-8911-7202.

${ }^{3}$ Licenciada en Comercio, Universidad Pedagógica Libertador. Doctora en Educación, Universidad Dr. Rafael Belloso Chacín. ORCID: https://orcid.org/0000-0002-8134-2306.
} 


\title{
Corporate environment in sustainable entreprenership
}

\begin{abstract}
This paper aims at analyzing corporate environment in sustainable entrepreneurship so that the influence of corporate environment in the development of entrepreneurship and its success could be identfied. This study focuses on the importance that corporate environment has in relation with social interaction. Different points of view from Druker (2011), Chirinos \& Perez (2016), Alcover, Rico \& Gil (2011), among others, were analyzed. This research was conducted from a quantitative paradigm; its orientation was descriptive and field transactional. The outcomes show that corporate environment is low, afecting the development of sustainable entrepreneurship in a negative way. To conclude, workers feel really satisfied with the corporate environment of their work areas..
\end{abstract}

Key words: corporate environment, intellectual capital, sustainable entrepreneurship.

\section{Climat organisationnel de l'entrepreneuriat responsable}

Résumé. L'objectif de cette étude est d'analyser le climat organisationnel de l'entrepreneuriat responsable afin d'identifier l'influence de l'environnement de travail sur le développement et le succès de l'entrepreneuriat et son importance dans l'interaction des individus. Les approches théroriques de Drucker (2011), Chirinos et Pérez (2016), Alcóver, Rico et Gil (2011) ont entre autres été utilisées. L'étude a été réalisée selon un paradigme quantitatif de type descriptif et transactionnel. Les résultats indiquent que le climat organisationnel se trouve globalement dégradé et qu'il impacte négativement le développement responsable de l'esprit d'entreprise, les employés n'étant généralement pas satisfaits du climat organisationnel dans leur travail.

Mots clefs: climat organisationnel, capital intellectuel, entrepreneuriat responsable.

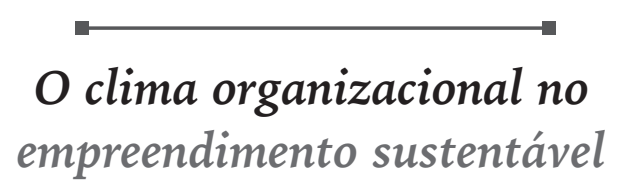

Resumo. O estudo tem como objetivo analisar o clima organizacional no empreendimento sustentável, com o fim de identificar a influência do ambiente laboral no desenvolvimento do empreendimento e no sucesso do mesmo, onde se faz ênfase na importância da interação entre as pessoas. Consideraram-se os enfoques de Drucker (2011), Chirinos \& Pérez (2016), Alcóver, Rico \& Gil (2011), entre outros. A pesquisa foi orientada a partir do paradigma quantitativo, o tipo de pesquisa foi descritiva, transacional de campo. Os resultados obtidos indicam que o clima organizacionalébaixo, incidindo negativamente no desenvolvimento do empreendimento sustentável. Em conclusão, os empregados se sentem insatisfeito com o clima organizacional em sua área laboral.

Palavras-chave: clima organizacional, capital Intelectual, empreendimento sustentável. 


\section{Introducción}

En la actualidad, el mundo empresarial $\mathcal{E}_{\text {se encuentra sometido por los cambios }}$ vertiginosos e inesperados de orden social, político, tecnológico, económico y cultural. El talento humano como factor social mantiene un intenso proceso de renovación cuyos elementos transformadores presentes en el proceso de modernización influyen considerablemente en la convivencia, las relaciones interpersonales y la participación de actividades y en el ambiente organizacional de las empresas tanto públicas como privadas.

De este modo, se hace énfasis en la importancia que tiene para las organizaciones la interacción entre las personas, la percepción del ambiente en el cual se desenvuelven para satisfacer sus necesidades básicas, entre ellas, actualización, ambiente de trabajo, salario, beneficios. Al respecto, Jones y James (1979) resaltan que toda organización debe tener un sentido de propósito, reconocimiento, compartir valores, oportunidades de aprendizaje y crecimiento, para fortalecer los procesos administrativos y el talento humano, que permita incrementar la eficiencia y efectividad de la organización.

En tal sentido, Rojas, Chirinos y Garcés (2017) indican que para lograr un buen clima organizacional es necesario estimar dentro de la estructura del emprendimiento la gestión de talento humano. Esta figura desempeña un papel fundamental que radica en ayudar a la organización a alcanzar sus objetivos, realizar su misión, hacerla competitiva, captar empleados idóneos, entrenarlos, motivarlos, facilitar el aumento de su autorrealización y satisfacción en el trabajo, desarrollar y mantener su calidad de vida, orientar su comportamiento a ser socialmente responsables, administrar el cambio y establecer políticas éticas. Esto se logra a partir de un proceso de perfeccionamiento de cada uno de los procesos y subprocesos que constituyen esta acción gerencial.

En este orden de ideas, según Chirinos (2008), un buen clima organizacional permite describir el conjunto de percepciones y expectativas de las personas que laboran en una organización y sus relaciones de trabajo, el cual facilita el diagnóstico del comportamiento organizacional, genera herramientas que hacen posible mitigar la fatiga que pueda presentarse en los trabajadores, propicia la intervención directa en las fallas y garantiza un buen clima laboral en la iniciativa de negocio.

Por otro lado, Chiang, Núñez, Martín y Salazar (2010) expresan que el clima organizacional son representaciones cognitivas del individuo, de eventos situacionales relativamente cercanos, que reflejan el significado psicológico y la significación de la situación para el individuo; estas percepciones se cree que son una función de componentes históricos, a saber, esquemas cognitivos que reflejan experiencias idiosincrásicas de aprendizaje. El ambiente laboral imperante en las organizaciones a nivel mundial influye directamente en las actitudes y el comportamiento de los trabajadores. Por tanto, las iniciativas de negocios se preocupan cada día por mantener un clima organizacional sano que fomente el desarrollo eficiente de los empleados para alcanzar los objetivos organizacionales.

Por tal motivo, el clima organizacional es una cualidad del ambiente laboral percibido y experimentado por los miembros de la organización, que es determinado por el líder. Las percepciones de los empleados 
de su entorno se reflejan en su naturaleza psicológica abstracta de cómo organizan las experiencias de su entorno; para cada individuo la percepción del entorno es diferente (Gibson, Donnelly, Ivancevich y Konopaske, 2011). Desde esta perspectiva, el clima organizacional influye directamente en los emprendimientos, ya que ellos están conformados por personas con deseos, aspiraciones y objetivos particulares que deben ser satisfechos y compartidos en el ambiente laboral en que se desarrollan. En tal sentido Hidrobo, Zapata, Vélez y González (2010) definen el emprendimiento como una forma de pensar, razonar y actuar orientada hacia la creación de riqueza, centrada en las oportunidades, planteada con visión global y llevada a cabo mediante un liderazgo equilibrado; es la gestión de un riesgo calculado, y su resultado es la creación de valor que beneficia a la empresa, la economía y la sociedad.

Uno de los mayores retos que enfrentan las iniciativas de negocio para alcanzar la sostenibilidad es lograr reunir un equipo de personas con la disposición para cumplir con el propósito primordial que tiene toda actividad empresarial como es ser efectivos y eficaces y los demás objetivos que se establezcan dependiendo de la particularidad del emprendimiento. Si bien es cierto que todas ellas posen necesidades, intereses, conocimientos, experiencias, expectativas y una serie de motivaciones diferentes, la función de la gestión del capital humano es consolidar esta variedad de destrezas para el bien común y de ese modo fusionarlas y hacerlas compartir el mismo fin, todo soportado en un mecanismo de comunicación fluido que llegue a todas las fibras del grupo y asó logre un excelente clima organizacional que conducirá al éxito del emprendimiento sostenible.
En tal sentido, Bernal, Pedraza y Sánchez, (2015) afirman que un gran número de organizaciones se encuentran en crisis, debido a su incapacidad para reaccionar adecuadamente a los cambios del medio donde operan. Las empresas que desean elevar su desempeño orientándose hacia la calidad deben buscar estrategias internas que las ayuden a alcanzar sus objetivos. Para satisfacer las complejas necesidades de los clientes y de sus trabajadores, se requiere un trabajo no individualizado; por el contrario, del uso exitoso de equipos, cuyos miembros tengan diferentes habilidades, relaciones y experiencias, incrementan la posibilidad de ofrecer soluciones creativas.

Sin embargo, un factor muy importante para que las empresas alcancen ser efectivas y eficaces es aprovechar sus recursos tecnológicos, financieros y humanos, a fin de crear ambientes de trabajo agradable y ventaja competitiva. En efecto, todos estos factores son variables que deben operar en conjunto con el fin de lograr los objetivos organizacionales previamente establecidos (Edvinsson y Malone, 2000).

En virtud de lo antes planteado, estos emprendimientos deben estar permanentemente atentos ante los cambios y las fluctuaciones provenientes del entorno que puedan afectar el desempeño del capital humano con que se cuenta y también poseer un estilo de gerencia innovador y equilibrado para posibilitar la sintonía de esta con el mercado tanto local como global. Por tanto, es necesario direccionar el capital humano hacia la productividad, sin dejar de lado la importancia de este para el logro del éxito y desarrollo del emprendimiento sostenible.

Por esto, es necesario comprender la importancia del clima organizacional como componente para el desarrollo del emprendimiento sostenible, puesto que permitirá el desarrollo 
fluido de ideas y la cooperación entre diversas personas con diferentes puntos de vista que complementen el trabajo y faciliten las funciones en las iniciativas de negocio, con la finalidad de alcanzar el éxito organizacional. Aquí radica la capacidad de integrar a los trabajadores en sus proyectos empresariales, de forma que todos consideren los objetivos corporativos colectivamente. $\mathrm{Y}$ es que las personas ya no son el activo más importante de la organización, sino que son la organización misma (Rodríguez et al., 2010).

Una vez realizada la revisión documental, según los planteamientos de Chiang et al. (2010), Drucker (2011), Chirinos y Pérez (2016), Alcover, Rico y Gil (2011), entre otros, los cuales proveen de argumentación teórica al presente estudio, se establecen comparaciones de la realidad y se llevan a cabo análisis interpretativos. Así, esta investigación se estructuró de la siguiente manera: fundamentación teórica, aspectos metodológicos, resultados y conclusiones finales.

Este trabajo se justifica porque busca generar insumos teóricos prácticos para crear y enfrentar acertadamente la dinámica del clima organizacional en el emprendimiento sostenible; y presenta alternativas, recursos y herramientas a estos, lo cual permitirá conocer el sentir de sus empleados en el área laboral. Por tanto, es primordial generar en el emprendimiento un ambiente agradable de trabajo, ya que permitirá el logro de sus objetivos, incrementará la productividad, hará a los empleados más efectivos y eficientes, conducirá al éxito organizacional y logrará la permanencia de estos en el mercado.

\section{Fundamentación teórica}

\subsection{Clima organizacional}

Según Peña, Díaz y Carrillo (2015), el clima organizacional en la actualidad es de interés para casi todas las empresas -familiares, emprendimientos, microempresas o PyMEs-, por su influencia en los procesos organizacionales y psicológicos, como la comunicación, la toma de decisiones, la solución de problemas, el aprendizaje, la motivación, y su repercusión en la eficiencia de la organización y en la satisfacción de sus miembros. Por ello, es necesario que estas se encuentren en óptimas condiciones desde el interior, donde exista satisfacción de su personal, de manera que impacte en la productividad. Hoy en día, los dirigentes empresariales han acrecentado el interés por establecer buenas interrelaciones bajo un clima organizacional que permita motivar al personal y así aumentar el desempeño, el interés y la satisfacción en el trabajo; es una ventaja para las organizaciones que el trabajador cuente con los elementos necesarios para desempeñarse adecuadamente, conservando y logrando la satisfacción, ya que los trabajadores satisfechos tienden a ser más cooperadores, adaptables y dispuestos al cambio.

El ambiente laboral influye enormemente en el comportamiento de los empleados, es decir, en la ejecución y el rendimiento de su trabajo. Por esta razón, la empresa debe conocer qué influye en este comportamiento, cuál es la percepción que tienen del ambiente laboral, de sus jefes, entre otros factores. La necesidad de conocer el funcionamiento de las organizaciones

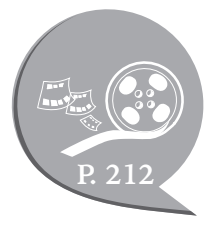


lleva implícito la descripción del ambiente donde se desarrolla la actividad de los individuos. Este ambiente en el trabajo puede describirse a través del clima organizacional, el cual es uno de los factores que más influye en el rendimiento laboral de los trabajadores de cualquier empresa, además en la propia calidad tanto de los productos elaborados como de los servicios prestados.

De tal modo, desde la perspectiva de Chirinos, Martínez y Meriño (2016), una compañía involucra una serie de objetivos, expectativas $\mathrm{y}$ obligaciones humanas que tienen como finalidad la producción de un bien o la prestación de un servicio para obtener una contrapartida de cualquier especie. Por tanto, en una organización, la supervivencia depende de la rentabilidad económica, y puede ser entendida como una comunidad especializada en la creación y transferencia rápida y eficiente de conocimiento, ya que por medio de su creación y uso, la empresa aprende, descubre, innova y puede adaptarse al cambio.

Al respecto, Chiang, Núñez, Martín y Salazar (2010) definen el clima organizacional como las características que distinguen una organización de otra, las cuales perduran a lo largo del tiempo e influyen en el comportamiento de las personas en las organizaciones. De esto se infiere que debe existir un buen ambiente laboral para propiciar el mejoramiento del desempeño de los trabajadores. En ese sentido, Campbell, Serfaty-de Medeiros y Viceira (2010) consideran que el clima organizacional se puede definir como un conjunto de atributos específicos de una organización en particular que puede ser inducido por el modo como la organización se enfrenta con sus miembros y su entorno en general. Para el miembro en particular dentro de la organización, el clima organizacional toma la forma de un conjunto de actitudes y expectativas que describen la organización respecto de características estáticas como grado de autonomía.

De la anterior definición se infiere que el clima organizacional es considerado como una variable situacional, la cual coincide con la de Hellriegel, Jackson y Slocum (2008), quienes indican que se refiere a una serie de atributos percibidos de una empresa o sus subsistemas y deducirse del modo en que la institución y sus subsistemas se relacionan con sus miembros y ambiente. Ahora bien, visto de esa forma, el clima organizacional describe los procesos comportamentales característicos de un sistema social de forma puntual. Estos conforman un conglomerado de actitudes y conductas que caracterizan la vida en la organización. Por tanto, es un atributo de la organización externo al individuo.

En el contexto anterior, se refleja que el clima organizacional constituye una configuración de las características de una organización y las del estilo gerencial, las cuales pueden constituir su personalidad interna. Bajo esa perspectiva, el clima organizacional es una cualidad o propiedad del ambiente laboral percibido y experimentado por los miembros de la empresa, determinada por el líder. Por consiguiente, se indica que el clima organizacional es un fenómeno circular en el que los resultados producidos vienen a confirmar las percepciones de los empleados. En otras palabras, las características psicológicas personales de estos, como actitudes, percepciones, personalidad, resistencia a la presión, valores y nivel de aprendizaje, sirven para interpretar la realidad circundante, las cuales se ven afectadas por los resultados obtenidos por la compañía.

Según los planteamientos anteriores, se concibe el clima organizacional como un componente multidimensional de elementos que 
pueden descomponerse respecto de estructuras organizacionales, tamaño de la empresa, modos de comunicación, estilos de liderazgo de la dirección, entre otros, lo cuales conforman un clima particular donde prevalecen sus propias características, le dan personalidad al emprendimiento e influyen en el comportamiento de los actores interactuantes en este.

\subsection{Emprendimiento sostenible}

Drucker (2011) plantea como tesis que solo aquellos emprendedores que tengan la capacidad de entender que la responsabilidad social de las empresas pasa por satisfacer de manera rentable las necesidades sociales de la población son los que están en condiciones de prosperar en el ámbito de los negocios. Desde el punto de vista de la investigación social, el emprendimiento sostenible es identificado como el proceso del estudio de la forma en la que son descubiertas creadas, ordenadas y explotadas las oportunidades, para traer a la existencia futuros bienes y servicios teniendo en cuenta sus consecuencias económicas, sociales y medioambientales.

En este contexto, el emprendimiento sostenible se caracteriza por ser una forma de integración social, humana y económica, con la finalidad de realizar iniciativas de negocios que permitan el aprovechamiento de las oportunidades que se presentan en el entorno. Así mismo, se identifica por buscar la mejor forma de producir bienes y servicios que satisfagan las necesidades de la colectividad e incorporen a su actividad la creatividad y la innovación. Otra de sus características relevantes es tomar al individuo como valor agregado de coopetencia para el desarrollo integral del entorno económico, y así lograr una estabilidad que garantice su sostenibilidad (Chirinos y Pérez, 2016).
En síntesis, el emprendimiento sostenible es un fenómeno deseable para la creación de bienes y servicios que satisfagan las necesidades de las personas, y que se produzca lo que realmente requieren las comunidades; por ello, es tan importante la planificación, la organización, la dirección y el control de las actividades que se desean realizar para desarrollar iniciativas de negocios, que vayan en beneficio del colectivo tanto al interior del emprendimiento como al exterior de este (Pérez, Chirinos y Martínez, 2017).

\subsection{Importancia del clima organizacional en el emprendimiento sostenible}

En el transcurrir de los años, el clima organizacional se ha convertido en un componente fundamental en los emprendimientos para el desarrollo de las actividades de las personas que la integran, lo cual repercute directamente en su productividad de forma positiva o negativa, todo va a depender de cómo se sientan los trabajadores en el área laboral y de la percepción que tengan de su entorno. Así es como un ambiente laboral agradable, armonioso y tranquilo permitirá el mejor desempeño de los empleados, quienes son de suma importancia para el emprendimiento ya que gran parte del éxito o fracaso de este es consecuencia del compromiso, las habilidades, el conocimiento y el aporte de ellos. Pero para hablar de compromiso, el emprendimiento, la organización o la empresa tienen que brindar a las personas espacios de participación, lugares donde puedan aportar su creatividad, su energía y su buen hacer, puesto que son el recurso estratégico de esta.

En el mismo orden de ideas, se manifiesta que en el cuidar de ese recurso está la clave de cualquier compromiso con la organización. El 
medio ambiente interno o clima organizacional de los emprendimientos tiene la obligación ética de ser un espacio de desarrollo y crecimiento de las personas que lo integran, que en su seno encuentren motivación y satisfacción a muchas de sus necesidades personales, ya que esto influye notoriamente en su comportamiento y desempeño. Por ello, el diseño estructural de las organizaciones, las políticas, las reglas, los procedimientos y los controles que enmarcan el desenvolvimiento: de las personas y sus actividades en los diferentes cargos deben tener condiciones de flexibilidad y adaptabilidad, por tal motivo es relevante que se cuente con un diseño eficaz, el cual debe caracterizarse por su consistencia y previsibilidad y por su sensibilidad y posibilidad de adaptación a los diferentes cambios.

Por su parte, García (2009) menciona que el clima organizacional es importante, ya que permite obtener información sobre el sentir de los colaboradores frente a los elementos que conforman la organización, como la metodología de trabajo, la estructura de la organización, el grado de supervisión por parte de los superiores, entre otros, de este modo, permite tener conocimiento de las condiciones laborales existentes, de los mecanismos de comunicación y de la relación laboral que existe. Así mismo, sostiene que es necesario el estudio del clima organizacional, puesto que permitirá conocer las percepciones de los diferentes elementos organizacionales por parte de los trabajadores, elementos que pueden ser políticas de la empresa, estructura organizativa, condiciones económicas de la empresa, diferentes estilos de liderazgo y valores que se perciben en la organización.

Por tanto, para entender el clima de una organización, es preciso comprender el comportamiento de las personas, la estructura de la organización y los procesos organizacionales, debido a que proporcionan información importante que sirve para mejorar las relaciones entre los equipos de trabajo y facilitar la toma de decisiones y el flujo de los procesos operativos de los emprendimientos en forma efectiva y eficiente. Así mismo, como lo plantean Meriño, Chirinos, Donawa y Martínez (2018), es importante considerar la calidad de vida en el trabajo, ya que representa la satisfacción generada por la idoneidad de las tareas, la cultura y el clima organizacional, el espíritu de equipo y de compañerismo, las percepciones salariales $y$ prestaciones, entre otros factores.

\subsection{Tipos de clima organizacional que influyen en el desarrollo del emprendimiento sostenible}

En lo que respecta a los tipos de clima organizacional, existe toda una serie de diferencias perceptuales, incluso cuando los individuos refieren aparentemente el mismo nivel descriptivo. Este puede concebirse entonces como el resultante de la interacción de las variables causales, intermedias y finales, la cual origina los diversos tipos de clima. En tal sentido, es importante destacar que las personas son el elemento más influyente en todas las organizaciones, son las encargadas de diseñar objetivos y estrategias, crean las innovaciones y desarrollan las actividades por las cuales una empresa logra la consolidación en el mercado; de ellas depende el triunfo o fracaso del emprendimiento sostenible (Rojas, Chirinos y Garcés, 2017).

De tal manera, es importante mantener un ambiente agradable en el cual las personas puedan desarrollar sus actividades de forma armoniosa y confortable; igualmente, que se compartan los objetivos de la empresa con los personales, hecho que motiva e induce a los trabajadores a rendir de forma efectiva y eficiente en sus labores, lo cual traerá como consecuencia mayor productividad 
y rentabilidad al emprendimiento. Por tal motivo, es relevante destacar que el clima de las organizaciones se ha clasificado en tipos diferentes. Alcover, Rico y Gil (2011) identifican tres niveles específicos, que se describen a continuación.

\subsubsection{Tipo psicológico}

El clima psicológico se ubica en el nivel individual y está constituido por las percepciones que los individuos tienen de su entorno, las cuales son de naturaleza psicológica, abstracta, no son descripciones de la realidad y reflejan cómo los individuos organizan las experiencias de su entorno. Así mismo, Clissold, Buttigieg y De Cieri (2012) indican que dichas percepciones individuales están relacionadas con eventos, prácticas y procesos de su ambiente de trabajo, incluso las percepciones conductuales que son premiadas, respaldadas en ese ambiente.

Gibson, Donnelly, Ivancevich y Konopaske (2011) señalan que estas no tienen por qué coincidir con las de otros individuos de su entorno, porque este es próximo de cada individuo, por tanto, es único, además, las diferencias individuales del trabajador desempeñan un importante papel. Este tipo de clima posee una débil diferenciación del estilo cognitivo del individuo, definido como la manera en la cual este organiza conceptualmente el entorno. Al respecto, los autores mencionados indican que el estilo cognitivo es un constructo desarrollado para explicar el proceso mediador entre el estímulo y la respuesta. Sin embargo, los psicólogos cognitivos sitúan la estructura sobre el contenido del pensamiento; en ese sentido, este tipo de clima se asocia con las conductas de las personas de manera independiente.

\subsubsection{Tipo agregado}

Se considera el tipo de clima agregado como el cálculo medio de las percepciones individuales de los grupos establecidos en las organizaciones, por ejemplo, el grupo de trabajo, el departamento, la división, la planta y la organización. En esa línea de pensamiento, considerando los postulados de Dessler (2008), quien indica que el nivel de agregación no necesita coordinarse con el nivel descriptivo de las percepciones, pero sí de un cierto nivel de consenso entre los miembros del grupo, este tipo de clima está basado en los miembros de las unidades formales de la organización y con acuerdo o consenso en las percepciones individuales, también se fundamenta en observaciones empíricas entre diferentes unidades, las cuales son atribuidas a discrepancias reales, y se relaciona con el nivel social, el cual permite hacer las cosas mediante otras personas e introducir las ideas de cohesión, liderazgo y poder (Alcover et al., 2011).

\subsubsection{Tipo colectivo}

El clima colectivo puede predecir las actitudes y el desempeño del personal de una organización, por tanto, se ubican en el nivel organizacional referido, según Alcover et al. (2011), al proyecto empresarial, el cual está determinado por una serie de factores sociales, tecnológicos, y financieros, que en conjunto determinan los límites de la empresa, tales como el sistema de remuneración, la comunicación, las relaciones interpersonales y la toma de decisiones, reflejados como se describe a continuación:

- Sistema de remuneración. Es uno de los mayores incentivos que tiene el personal en el momento de realizar sus actividades; todas las personas que desempeñan sus labores esperan por ello una retribución 
justa: salario, bonos, seguros de vida y salud, entre otros, que los motive a contribuir con el desarrollo de la institución (Rojas, Chirinos y Garcés, 2017).

En tal sentido, Chiavenato (2011) indica que, como socio de una organización, cada empleado se interesa en invertir trabajo, dedicación y esfuerzo personal, conocimientos y habilidades, y así recibir la retribución y las compensaciones adecuadas que lo hacen sentir satisfecho y lo motivan a ser cada vez más eficiente en su labor. Así mismo, las organizaciones se interesan en invertir en compensación para las personas, ya que reciben contribuciones que estén al alcance de sus objetivos.

- Comunicación. Es la percepción general sobre el estado de comunicación y capacidad de escuchar por parte de la empresa que determina el ambiente laboral; es importante considerarla como elemento clave de este proceso de interacción, ya que a través de esta se establecen las relaciones humanas y de trabajo y se definen parámetros de competitividad y productividad laboral.

- Relaciones interpersonales. Yañez, Arenas y Ripoll (2010) señalan que este aspecto se corresponde con la relación entre un grupo o conjunto de personas que desarrollan una actividad o evento determinado en concordancia con la normativa establecida para tal relación. Robbins y Coulter (2010) las asocian con la percepción por parte de los miembros de la organización acerca de la existencia de un ambiente de trabajo grato y de buenas relaciones sociales, tanto entre pares como entre jefes y subordinados, generadas, Así mismo, dentro como fuera de la institución.
- Toma de decisiones. Es valorada como una de las tareas más críticas que enfrenta un gerente, ya que sus decisiones giran en torno a las principales funciones que se espera desempeñe como líder, entre otras, definición de objetivos, determinación de medios para lograr las metas, defensa de la empresa de amenazas externas, solución de conflictos internos. Por otra parte, Rodríguez et al. (2010) destacan que esta en una organización se circunscribe a una serie de personas que están apoyando el mismo proyecto. Por esta razón, se debe empezar por hacer una selección de alternativas, que se ha de convertir en una de las tareas de gran trascendencia, la cual requiere del empleo de un buen juicio donde un problema o situación es valorado y considerado profundamente para la elección del mejor camino a seguir en función de las diferentes alternativas posibles.

\subsection{Factores del clima organizacional que inciden en el desarrollo del emprendimiento sostenible}

El individuo percibe el clima organizacional solo en función de las necesidades que el emprendimiento le puede satisfacer; para ello, es importante que la organización considere en su interior la formación profesional en relación con sus procesos de capacitación como un elemento de valor agregado para el personal, lo cual permitirá el desarrollo de competencias en el área laboral como habilidades, conocimientos, actitudes, valores, comunicación efectiva, trabajo en equipo, entre otros (Chirinos, Pérez y Pachón 2017). En tal sentido, Chiavenato (2011) indica que el clima dentro de una organización depende de la interacción entre factores externos, tales como ambiente organizacional, tamaño de la 
organización, estructura organizativa, entre otros; y de internos, motivación, comunicación, toma de decisiones, trabajo en equipo.

\subsubsection{Factores internos}

Los factores internos del clima organizacional, según Gibson et al. (2011), se refieren al comportamiento de los empleados de una empresa, quienes se caracterizan por actitudes, percepciones, personalidad, estrés, valores y aprendizajes propios del individuo que los diferencian de los demás y les permiten desempeñarse en la empresa. A continuación, se describen los factores internos del clima organizacional que tienen incidencia en el emprendimiento sostenible.

- Motivación. En el contexto organizacional, la motivación representa aquellos procesos psicológicos que causan la estimulación, dirección y persistencia de acciones voluntarias dirigidas a los objetivos organizacionales. Según Robbins y Coulter (2010), se describe como los procesos responsables del deseo de un individuo de realizar un gran esfuerzo para lograr los objetivos organizacionales, condicionado por la capacidad del esfuerzo de satisfacer alguna necesidad individual. Así mismo, Chiavenato (2011) la considera como aquello que impulsa a una persona a actuar de determinada manera o por lo menos, que origina una propensión a un comportamiento específico. En estas definiciones, se evidencia la motivación como uno de los factores internos principales de toda organización, que tiene en cuenta las necesidades de los empleados y el efecto de esta en su comportamiento o desempeño en el trabajo.
- Comunicación. Una idea, no importa cuán magnífica sea, no sirve de nada si no logra ser transmitida y entendida por otros. Es decir, es necesario que la apreciación mental del emisor sea igual que la del receptor para que la comunicación sea perfecta, lo cual en la realidad nunca se logra, entre otras cosas, por la percepción que cada individuo tiene del mundo en el que se desarrolla, de ahí que la comunicación o mejor, la mala comunicación, sea el motivo más citado de los conflictos entre las personas. Por consiguiente, según Robbins \& Coulter (2010), se considera la comunicación como la transferencia y el entendimiento del significado y según el nivel de una organización, sirve para ejercer control, para motivar a las personas, para informar y como alivio a la expresión emocional de los sentimientos; como elemento interno, facilita las relaciones interpersonales en la organización, por cuanto implica transferencia de información y significado de una persona a otra. En ese sentido, la comunicación hace que las personas se relacionen con sus semejantes por medio de símbolos los cuales conducen a una comunicación más efectiva y el mensaje llega más rápido, aun sin obstáculos.

- Toma de decisiones. Según Koontz y Weihrich (2012), es el núcleo de la planeación, y se define como la selección de un curso de acción entre varias alternativas, no puede decirse que exista un plan a menos que se haya tomado una decisión, que se hayan comprometido los recursos, la dirección o la reputación; hasta ese momento solo existen estudios de planeación y análisis. Algunas veces, los gerentes consideran que la toma de decisiones es su principal tarea, pues constantemente deciden qué hacer, quién debe

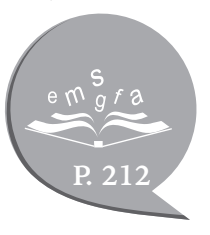


hacerlo y cuándo, dónde, incluso cómo se ha de hacer; sin embargo, la toma de decisiones es solo un paso en el sistema de planeación. No obstante, en toda organización, además de las decisiones normales que se toman día a día, también deben tomarse decisiones por medio de planes de contingencia, los cuales no son más que actividades planificadas para dar solución a un problema suscitado por algún evento externo influyente del ambiente interno de la organización.

- Trabajo en equipo. Es un factor que permite la integración de los integrantes de la organización para darles a conocer sus objetivos y que contribuyan de manera responsable y entusiasta a la resolución de tareas, apoyándose entre sí, hecho que es esencial en las organizaciones por cuanto los empleados deben formar grupos para realizar las tareas asignadas. En tal sentido, se describe como un estilo para realizar una actividad laboral, es asumir un conjunto de valores y un espíritu que anima a un modelo de relaciones entre las personas y un modo de participación plena en el trabajo basado en la confianza, la comunicación, la sinceridad y el apoyo mutuo, donde se privilegia la interdependencia activa, consciente y responsable de sus miembros, lo cual les induce asumir la misión del equipo como propia (Alcover et al., 2011).

\subsubsection{Factores externos}

Los factores externos, según Chiavenato (2011), se refieren a la realidad de las organizaciones, las cuales son visibles por cuanto de ellas depende el desempeño de su personal y su productividad. Por ello, los constantes cambios dados a nivel económico, administrativo y tecnológico explican el clima existente en una determinada organización. Entre ellos, están el ambiente organizacional, el tamaño de la organización y la estructura organizativa, tal como se menciona a continuación.

- Ambiente organizacional. Se refiere al contexto que involucra a la organización, puede ser también la situación dentro de la cual una empresa se encuentra insertada y le permite mantener transacciones e intercambio con su ambiente. Este factor afecta el rendimiento de la compañía e influye directamente en su comportamiento, por ello, si el ambiente externo es complejo a la institución empresarial, le costará mucho adaptarlo a su ambiente interno de trabajo. A tal respecto, establece como un elemento clave del ambiente la identidad organizativa, la cual es el grado en que el empleado se identifica con la organización, sus valores, costumbres, el sentimiento de pertenencia y produce mejoras en el grupo de trabajo.

- Infraestructura de la organización. Se refiere a las condiciones básicas que permiten el curso del trabajo en una organización, espacio razonable en un edificio equipado con luz adecuada, agua limpia y una fuente confiable de electricidad y transporte viable para el traslado de los empleados. Dentro de esta infraestructura, se ubica la planta física, concebida como las áreas físicas necesarias para realizar las actividades propias de la organización. De igual forma es un elemento importante del clima organizacional, porque, si la organización no posee una planta física adecuada a las necesidades del personal, este no se verá motivado, por tanto, no podrá tener sentido de pertenencia hacia su organización. 
- Estructura organizativa. Es la representación formal de las relaciones laborales, define las tareas, los puestos y la unidad, señala que esta es la manera de coordinarse, cubre las directrices, consignas y políticas que puede emitir una organización y que afectan directamente la forma de llevar a cabo una determinada tarea (Brunet, 2009). Así mismo, Robbins y Coulter (2010) afirman que en ella se agrupan y coordinan formalmente las tareas en el trabajo. Toda empresa para poder funcionar debe tener o poseer un organigrama con el cual se establecen las funciones de cada uno de sus miembros, de igual forma se elabora la visión y misión de la empresa. De acuerdo con lo antes planteado, en toda organización debe existir una estructura organizativa, la cual detalla los cargos y las funciones de cada persona que labora en ella, tomando en consideración las funciones desempeñadas por cada miembro del personal. Dentro de ella, se establecen las cadenas de mando y el poder.

\section{Metodología}

$\mathrm{E}_{\mathrm{c} a}^{\mathrm{s}}$ importante señalar que metodológicamente la investigación se ubica en el racionalismo científico como corriente epistemológica fundamental, la cual advierte que la fuente de conocimientos reales está en el uso de procedimientos objetivos determinados por la razón, como fuente y fin de producción de conocimientos fiables. Tal como refiere Sabino (2014, p. 128), las proposiciones no son acumuladas desordenadamente, ni en simples formas cronológicas o jerárquicas, sino en conjuntos ordenados de enunciados que aspiran a formar sistemas teóricos congruentes. En tal sentido, el presente estudio está orientado bajo el paradigma cuantitativo, y el tipo de investigación fue descriptivo y transeccional de campo.

\subsection{Población}

El estudio está dirigido a la población de 63 sujetos, quienes conforman el personal que labora en la empresa de servicios y mantenimiento Baralt, ubicada en Mene Grande
-Zulia, Venezuela-; se toma esta empresa como referente de emprendimiento sostenible por sus características y mantenimiento en el mercado. Debido a lo pequeña de su población, se decide no extraer muestra ya que la cantidad de personas por estudiar es de fácil manipulación y acceso.

\subsection{Técnicas e instrumento de recolección de datos}

La recolección de datos se realizó a través del cuestionario autoadministrado, en un periodo de dos meses, durante el segundo semestre de 2016, bajo los parámetros de la escala de Likert -con cinco opciones de respuesta por cada aseveración planteada-, el cual se utilizó para recolectar información pertinente en relación con los objetivos de la investigación, que constituyó la manera más sencilla para la obtención de información.

Desde esta perspectiva, la recolección de la información se llevó a cabo mediante la aplicación de un cuestionario con 20 ítems 
y un escalamiento de cinco alternativas de respuestas -5: siempre; 4: casi siempre; 3 : algunas veces; 2 : casi nunca; 1 : nunca-, con el cual se logró analizar el clima organizacional en el emprendimiento sostenible.

\subsection{Baremo de interpretación de la media aritmética}

A continuación, se presenta la información de las categorías escogidas y las ponderaciones correspondientes para la interpretación de la media (Tabla 1).

Tabla 1. Baremo de interpretación de la media aritmética para el clima organizacional en el emprendimiento sostenible

\begin{tabular}{|c|c|}
\hline Rangos & Categorías \\
\hline $3.80-5.00$ & Alto \\
\hline $2.40-3.79$ & Medio \\
\hline $1.00-2.39$ & Bajo \\
\hline
\end{tabular}

Fuente. Elaboración propia.

\section{Resultados}

$\mathrm{D}^{\circ}$ acuerdo con lo expuesto un poco más adelante (tabla 2), con respecto al indicador tipo de clima organizacional psicológico, la muestra de los 63 empleados expresó que casi nunca con un $39.68 \%$ y nunca con un $31.75 \%$ se sienten a gusto en el ambiente de trabajo y no están satisfechos con el cargo que ocupan en la empresa.

La media del indicador tipo de clima organizacional psicológico es de 2.08 puntos. Según el baremo establecido, se sitúa en la categoría bajo, es decir, los empleados tienen un tipo de clima psicológico bajo en la empresa.

En referencia al indicador tipo de clima agregado, los resultados obtenidos por los empleados establecen que: algunas veces con un $23.81 \%$, casi nunca con un $6.35 \%$ y nunca con un $22.22 \%$ la gerencia se esfuerza con su equipo de trabajo para el logro de los objetivos organizacionales ni promueve trabajo en equipo dentro de la empresa.
Con respecto a la media del indicador tipo de clima agregado, es de 3.05 puntos, que se sitúa en la categoría media clima organizacional, según el baremo establecido, es decir, los empleados tienen un tipo de clima agregado medio en la empresa.

En cuanto al indicador tipo de clima colectivo, los valores obtenidos indican: algunas veces con un $4.76 \%$, casi nunca con un $63.49 \%$, y nunca con un $31.75 \%$ existe una comunicación efectiva entre personal directivo y el capital humano ni la gerencia toma en cuenta sus opiniones.

La media general del indicador tipo clima colectivo es de 1.73 puntos que corresponde a la categoría bajo, es decir, según el baremo señalado, los empleados tienen un clima agregado bajo en la empresa. 
En conclusión, la media general de la dimensión tipo de clima organizacional es de 2.29 puntos, que la sitúa en un tipo de clima bajo, es decir, según el baremo señalado, los empleados afirman que predominan en la empresa los tipos de climas psicológico, agregado y colectivo bajo, lo cual refleja que los trabajadores no se sienten cómodos en su ambiente laboral.

Tabla 2. Dimensión: tipos de clima organizacional que influyen en el emprendimiento sostenible

\begin{tabular}{|c|c|c|c|c|c|c|c|c|c|c|c|}
\hline \multirow[b]{2}{*}{ Indicador } & \multicolumn{2}{|c|}{ Siempre } & \multicolumn{2}{|c|}{ Casi siempre } & \multicolumn{2}{|c|}{$\begin{array}{l}\text { Algunas } \\
\text { veces }\end{array}$} & \multicolumn{2}{|c|}{ Casi nunca } & \multicolumn{2}{|c|}{ Nunca } & \multirow{2}{*}{$\begin{array}{l}\text { Total } \\
\text { Media }\end{array}$} \\
\hline & $\mathrm{fa}$ & $\%$ & $\mathrm{fa}$ & $\%$ & $\mathrm{fa}$ & $\%$ & $\mathrm{fa}$ & $\%$ & $\mathrm{fa}$ & $\%$ & \\
\hline Psicológico & 1 & 1.58 & 5 & 7.94 & 12 & 19.05 & 25 & 39.68 & 20 & 31.75 & 2.08 \\
\hline Agregado & 5 & 7.94 & 25 & 39.68 & 15 & 23.81 & 4 & 6.35 & 14 & 22.22 & 3.05 \\
\hline Colectivo & 0 & 0.00 & 0 & 0.00 & 3 & 4.76 & 40 & 63.49 & 20 & 31.75 & 1.73 \\
\hline Media & 2 & 3.17 & 10 & 15.87 & 10 & 15.87 & 23 & 36.51 & 18 & 28.57 & 2.29 \\
\hline
\end{tabular}

Fuente. Elaboración propia.

Con respecto al indicador factores internos del clima organizacional (Tabla 3), la muestra de los empleados expresó que casi nunca con un $47.62 \%$ y nunca con un $31.75 \%$ el personal gerencial está atento en la realización de las actividades asignadas y la gerencia, junto con su capital humano, resuelve los problemas encontrados en la ejecución de sus actividades para así darles solución en equipo.

En referencia a la media general del indicador factores internos del clima organizacional, que es de 2.10 puntos, según el baremo establecido, los factores internos del clima organizacional son bajos en la empresa.
En cuanto al indicador factor externo del clima organizacional, las muestras de los empleados expresaron que casi nunca con un $47.62 \%$ y nunca con un $15.87 \%$ sienten que el horario de trabajo dentro de la empresa les brinda comodidad a los empleados para que estos puedan realizar sus actividades y sienten que están identificados con la organización.

La media general del indicador factores externos del clima organizacional, es de 2.57 puntos, y establece que el factor externo del clima organizacional es mediano en la empresa, según el baremo señalado. Lo anterior que indica que la gerencia no considera importante el ambiente laboral de los empleados.

Tabla 3. Dimensión: factores del clima organizacional que inciden en el emprendimiento sostenible

\begin{tabular}{|c|c|c|c|c|c|c|c|c|c|c|c|}
\hline & \multicolumn{2}{|c|}{ Siempre } & \multicolumn{2}{c|}{ Casi siempre } & \multicolumn{2}{c|}{ Algunas veces } & \multicolumn{2}{c|}{ Casi nunca } & \multicolumn{2}{|c|}{ Nunca } & Total \\
\hline Indicador & $\mathrm{fa}$ & $\%$ & $\mathrm{fa}$ & $\%$ & $\mathrm{fa}$ & $\%$ & $\mathrm{fa}$ & $\%$ & $\mathrm{fa}$ & $\%$ & Media \\
\hline Internos & 0 & 0.00 & 13 & 20.63 & 0 & 0.00 & 30 & 47.62 & 20 & 31.75 & 2.10 \\
\hline Externos & 0 & 0.00 & 23 & 36.51 & 0 & 0.00 & 30 & 47.62 & 10 & 15.87 & 2.57 \\
\hline Media & 0 & 0.00 & 18 & 28.57 & 0 & 0.00 & 30 & 47.62 & 15 & 23.81 & 2.33 \\
\hline
\end{tabular}

Fuente. Elaboración propia. 
Con respecto a la dimensión tipos de clima organizacional, la muestra de los empleados expresó que casi nunca con un $36.51 \%$ y nunca con un $28.57 \%$ sienten un tipo de clima organizacional que los satisfaga en la organización, en tanto que en la media se identifica que los tipos de clima organizacional en la empresa se encuentran en la categoría bajo, según el baremo establecido con una media de 2.29 puntos.

Así mismo, en la dimensión factores del clima organizacional, la muestra de los empleados expresó que casi nunca con un $47.62 \%$ y nunca con un $23.81 \%$ sienten los factores del clima organizacional en la empresa, mientras que en referencia a la media, se identifica que los factores del clima organizacional en la empresa se encuentran en la categoría bajo según el baremo establecido con una media de 2.33 puntos.

Se puede evidenciar que la media general de la variable clima organizacional, que es de 2.31 puntos, indica la existencia de un clima organizacional bajo en la empresa según el baremo establecido, lo cual confirma que los empleados están insatisfechos con el clima organizacional de la empresa y se sienten desmotivados en el momento de desarrollar sus labores diarias.

Tabla 4. Clima organizacional en el emprendimiento sostenible

\begin{tabular}{|c|c|c|c|c|c|c|c|c|c|c|c|}
\hline & \multicolumn{2}{|c|}{ Siempre } & \multicolumn{2}{c|}{ Casi siempre } & \multicolumn{2}{c|}{ Algunas veces } & \multicolumn{2}{c|}{ Casi nunca } & \multicolumn{2}{|c|}{ Nunca } & Total \\
\hline Dimensiones & $\mathrm{fa}$ & $\%$ & $\mathrm{fa}$ & $\%$ & $\mathrm{fa}$ & $\%$ & $\mathrm{fa}$ & $\%$ & $\mathrm{fa}$ & $\%$ & Media \\
\hline Tipos & 2 & 3.17 & 10 & 15.87 & 10 & 15.87 & 23 & 36.51 & 18 & 28.57 & 2.29 \\
\hline Factores & 0 & 0.00 & 18 & 28.57 & 0 & 0.00 & 30 & 47.62 & 15 & 23.81 & 2.33 \\
\hline Media & 1 & 1.58 & 13 & 20.63 & 6 & 9.52 & 26 & 41.27 & 17 & 26.98 & 2.31 \\
\hline
\end{tabular}

Fuente. Elaboración propia.

\section{Consideraciones finales}

Según los resultados obtenidos, se logró Sidentificar que los empleados se sienten insatisfechos con relación al clima organizacional, ya que este indicador se muestra en nivel bajo con un porcentaje del $2.31 \%$ en el emprendimiento sostenible reflejado en la medición antes descrita. Por ello, la ausencia de un clima laboral agradable en la empresa afecta la estabilidad emocional de los empleados, de ahí la importancia de tener un ambiente laboral favorable en la organización al permitir comprender la conducta individual, el desarrollo de acciones encaminadas a alcanzar las metas y el sistema personal, puesto que si el personal se siente motivado, seguro y cómodo en el desarrollo de sus actividades, logra ser eficiente y eficaz en su desempeño lo cual conduce al logro de los objetivos de la empresa.

En tal sentido, los principios básicos de calidad se fundamentan en un clima organizacional como condición necesaria para conseguir aumentos de productividad y competitividad, referencia esta que es esencial. Se considera 
que, cuando las personas trabajan en una organización y se sienten contentas con la función desempeñada, el compromiso con esta aumenta y mejoran los resultados. Sin embargo, los resultados obtenidos en el presente estudio demuestran que en relación con los factores del clima organizacional que inciden en el emprendimiento sostenible, estos se encuentran en un nivel bajo, con un porcentaje del $2.33 \%$ según el baremos establecido para la interpretación de la media, lo cual afecta el desarrollo y éxito de este, pues los empleados no se sienten satisfechos, motivados, sienten la falta de comunicación entre ellos y el nivel gerencial; no están conformes con las decisiones que se toman en la organización, no están integrados con sus compañeros de trabajo, y por consiguiente, el trabajo en equipo es deficiente. Así mismo, el tipo de clima que influye en el emprendimiento sostenible también se ubicó en un nivel bajo, con un porcentaje del $2.29 \%$, lo cual demuestra que los trabajadores no se sienten cómodos en su ambiente laboral.

Por consiguiente, es importante considerar los factores del clima organizacional puesto que se refieren a los comportamientos de los empleados de una empresa caracterizados por las actitudes, las percepciones, la personalidad, el estrés, los valores y los aprendizajes propios del individuo, los cuales lo diferencian de los demás y les permiten desenvolverse dentro de la organización por medio de estos; entre ellos están la motivación, la comunicación, la toma de decisiones y el trabajo en equipo. Estos elementos permiten facilitar el ambiente laboral en los trabajadores y conducen a una mejor productividad para el emprendimiento sostenible, y por ende, un mejor desarrollo sustentable.

DE igual forma, la actividad organizacional se puede ver como un proceso complejo de conversión de recursos a través de un sistema integrado capaz de proporcionar salidas o resultados, proceso que requiere la combinación óptima de entradas -esfuerzo humano, tecnología, materias primas, capital, entre otros- junto con trabajo para producir el resultado esperado. Por consiguiente, uno de los objetivos principales de los emprendimientos es la generación de riquezas y la inclusión laboral, por lo cual los aspectos más importantes de la filosofía de una organización se relacionan con la política de retribución y compensación a los trabajadores; dado que ella representa un costo para las empresas, estas deben analizar la relación de los costos y beneficios de sus sistemas de remuneración.

En otros términos, los sistemas de compensación deben generar retornos para la organización, además de incentivar a las personas a contribuir en ella. Por esta razón, es de vital importancia mantener un clima organizacional agradable donde los empleados se sientas satisfechos y compartan los objetivos de la organización; adicionalmente, que estos los lleven a conseguir sus objetivos personales para satisfacer sus necesidades, lo cual garantiza alto desempeño dentro del emprendimiento sostenible y el éxito de este.

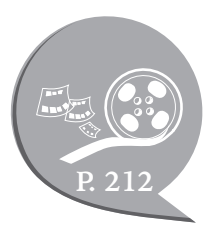




\section{Referencias}

Alcover, C. M., Rico, R. y Gil, F. (2011). Equipos de trabajo en contextos organizacionales: dinámicas de cambio, adaptación y aprendizaje en entornos flexibles. Papeles del Psicólogo, 32(1), 7-16.

Bernal González, I., Pedraza Melo, N. A. y Sánchez Limón, M. L. (2015). El clima organizacional y su relación con la calidad de los servicios públicos de salud: diseño de un modelo teórico. Estudios Gerenciales, 31(134), 8-19.

Brunet, L. (2009). El clima de trabajo en las organizaciones: definiciones, diagnóstico y consecuencias. México: Trillas.

Campbell, J. Y., Serfaty-De Medeiros, K. y Viceira, L. M. (2010). Global currency hedging. The Journal of Finance, 65(1), 87-121.

Castrillón Velásquez, D. A. (2011). Del líder al humano en la organización. Pensamiento y Gestión, 31, 34-55.

Cecchini, S. y Martínez, R. (2011). Protección social inclusiva en América Latina: una mirada integral, un enfoque de derechos. Santiago de Chile: Comisión Económica para América Latina y el Caribe.

Clissold, G., Buttigieg, D. M. y De Cieri, H. (2012). A psychological approach to occupational safety. Asia Pacific Journal of Human Resources, 50(1), 92-109.

Chiavenato, I. (2011). Comportamiento organizacional: la dinámica del éxito en las organizaciones (2. ${ }^{\mathrm{a}}$ ed.). México: Thomson.

Chiang, M., Núñez, A., Martín, M.J.y Salazar, M. (2010). Compromiso del trabajador hacia su organización y la relación con el clima organizacional: un análisis de género y edad. Panorama Socioeconómico, 28(40), 92-103.

Chirinos Araque, Y. del V. (2008). El clima de trabajo en las organizaciones. México: Trillas.

Chirinos Araque, Y. del V. y Pérez Peralta, C. M. (2016). La responsabilidad social universitaria: emprendimiento sostenible como impacto de intervención en comunidades vulnerables. Revista EAN, 81, 91-110.
Chirinos Araque, Y. del V., Martínez de Meriño, C. Y. y Meriño Córdoba, V. H. (2016). El emprendimiento sostenible como generador de conocimiento en las pymes. En Meriño Córdoba V. H.; Chirinos Araque, Y. del V.; Camejo López L. y Martínez de Meriño C. Y. (comps.). Gestión del conocimiento: perspectiva multidisciplinaria (vol. 1, pp. 36-8). Coro, Venezuela: Universidad Politécnica Territorial de Falcón Alonso Gamero.

Chirinos Araque, Y. del V., Pérez Peralta, C. M. y Pachón Flórez, C. M. (2017). Emprendimiento sostenible en las pymes para la generación de empleo. En Y. del V. Chirinos Araque, C. M. Pérez Peralta, M. C. Barrios Barreto y C. Y. Martínez de Meriño (comps.), Universidad, ciencia, innovación y sociedad: desde la perspectiva laboral (pp. 11-48). Barranquilla: Universidad Simón Bolívar.

Dessler, G. (2008). Administración de los recursos humanos. México: Pearson.

Drucker, P. F. (2011). Landmarks of tomorrow: A report on the new. New Brunswick: Transaction Publishers.

Edvinsson, L. y Malone, M. (2000). El capital intelectual. Barcelona: Gestión 2000.

García Solarte, M. (2009). Clima organizacional y su diagnóstico: una aproximación conceptual. Cuadernos de Administración, 42, 43-61.

Gibson, J. L., Donnelly, J. H., Ivancevich, J. M. y Konopaske, R. (2011). Organizaciones comportamiento, estructura y procesos (3. ${ }^{\mathrm{a}}$ ed.). México: McGraw-Hill.

Hellriegel, D., Jackson, S. y Slocum, J. (2008). Administración: un enfoque basado en competencias (11. ${ }^{\mathrm{a}}$ ed.). México: Cengage.

Hidrobo, L. C., Zapata Cruz, M., Vélez Zapata, D. M. y González Abadía, M. D. (2010). Plan de área emprendimiento. Recuperado de https://issuu.com/ dianacristinavalenciaquintero5/docs/47370039plan-de-area-de-emprendimi

Jones, A. P. y James, L. R. (1979). Psychological climate: Dimensions and relationships of individual and aggregated work environment perceptions. Organizational Behavior and Human Performance, 23 (2), 201-250. 
Koontz, H. y Weihrich, H. (2012). Administración una perspectiva global y empresarial. México: McGraw-Hill.

Meriño, V. Chirinos, Y. Donawa, Z. Martínez R. (2018). Calidad de vida laboral en el personal administrativo de la Universidad Nacional Experimental Rafael María Baralt. En V. H. Meriño Córdoba, Y. del V. Chirinos Araque, L. H. Camejo López y C. Y. Martínez de Meriño (comps.), Gestión del conocimiento: perspectiva multidisiplinaria (vol. 3, pp. 2-25). Coro, Venezuela: Universidad Politécnica Territorial de Falcón Alonso Gamero.

Peña Cárdenas, M. C., Díaz Díaz, M. G. y Carrillo Puente, A. (2015). Relación del clima organizacional y la satisfacción laboral en una pequeña empresa familiar. Revista Internacional Administración \& Finanzas, 8(1), 37-50. Recuperado de http://bit. ly/2IyhjUa

Pérez Peralta, C. M., Chirinos Araque, Y. del V. y Martínez de Meriño, C. Y. (2017). Emprendimiento social sostenible en comunidades vulnerables: caso Altos de la Sabana de la ciudad de Sincelejo, Sucre, Colombia. En Y. del V. Chirinos Araque, C. M. Pérez Peralta, M. C. Barrios Barreto y C. Y. Martínez de Meriño (comps.), Universidad, ciencia, innovación y sociedad: desde la perspectiva laboral (pp. 233-260). Barranquilla: Universidad Simón Bolívar.
Robbins, S. P. y Coulter, M. (2010). Administración (10. ed.). Madrid: Pearson.

Rodríguez Salvá, A., Álvarez Pérez, A., Sosa Lorenzo, I., De Vos, P., Bonet Gorbea, M. H. y Van der Stuyft, P. (2010). Inventario del clima organizacional como una herramienta necesaria para evaluar la calidad del trabajo. Revista Cubana de Higiene y Epidemiología, 48(2), 177-196.

Rojas Nieves, D. C., Chirinos Araque, Y. del V. y Garcés Mejías, G. del C. (2017). El nuevo reto de la gerencia del talento humano los empleados tóxicos. En V. H. Meriño Córdoba, Y. del V. Chirinos Araque, L. H. Camejo López y C. Y. Martínez de Meriño, Gestión del conocimiento: perspectiva multidisiplinaria (vol. 2, pp. 73-103). Coro, Venezuela: Universidad Politécnica Territorial de Falcón Alonso Gamero

Sabino, C. (2014). El proceso de investigación. Guatemala: Episteme.

Stephen, R. y Timothy, J. (2009). Comportamiento organizacional (13. ${ }^{\mathrm{a}}$ ed.). México: Pearson.

Yañez Gallardo, R., Arenas Carmona, M. y Ripoll Novales, M. (2010). El impacto de las relaciones interpersonales en la satisfacción laboral general. Liberabit, 16(2), 193-202. 